\title{
La méthode PICT (Pollution-Induced Community Tolerance) appliquée aux communautés algales : intérêt comme outil de diagnose et d'évaluation du risque écotoxicologique en milieu aquatique
}

\author{
A. Bérard ${ }^{1,4}$ \\ U. Dorigo ${ }^{2}$ \\ J.F. Humbert ${ }^{2}$ \\ C. Leboulanger ${ }^{2}$ \\ F. Seguin ${ }^{3}$
}

Mots-clés : ecotoxicologie, sélection, tolérance, algues

Pour caractériser l'état d'un écosystème aquatique contaminé par des toxiques il est nécessaire de développer des méthodes d'évaluation des impacts écotoxicologiques, basées à la fois sur l'étude de réponses physiologiques et sur la structure des communautés vivant dans cet écosystème. La méthode PICT (Pollution-Induced Community Tolerance) proposée par Blanck et al. (1988), répond à ces deux critères. Elle est fondée sur l'hypothèse qu'une communauté biologique naturelle est constituée de différents «composants» ayant des sensibilités variables vis-à-vis d'un toxique donné. Ainsi, suite à une exposition à un toxique, les organismes les plus sensibles ne sont plus concurrentiels et sont remplacés par des organismes plus tolérants. La communauté résultante présente alors une tolérance vis-à-vis du toxique, supérieure à celle observée pour une communauté semblable mais n'ayant pas connu de pression de sélection par ce toxique. Cette différence de réponse peut donner une indication sur l'état de pollution des milieux dans lesquels ont été échantillonnées différentes communautés. Le choix du test physiologique pour évaluer la tolérance de la communauté est fondamental car la réponse dépend d'une part, du mode d'action du toxique et d'autre part, des mécanismes à l'origine de la tolérance de la communauté. Il existe peu d'outils de bioévaluation des milieux naturels soumis à des contaminations toxiques, et cette méthode mérite donc d'être développée. Des travaux appliquant la méthode PICT à des communautés variées et dans divers milieux, permettraient de valider la méthode et de résoudre certains problèmes, quant à la détection de la tolérance. Le développement de méthodologies nouvelles pour caractériser la structure et la tolérance des communautés étudiées est fondamental pour progresser dans nos connaissances sur le risque écotoxicologique.

Application of the Pollution-Induced Community Tolerance (PICT) method to algal communities: its values as a diagnostic tool for ecotoxicological risk assessment in the aquatic environment

Keywords : ecotoxicology, selection, tolerance, algae.

Methodologies based on physiological responses and biological community structure, such as the Pollution-Induced Community Tolerance (PICT) proposed by Blank et al 1988, are required to characterise ecosystem health. The PICT concept is based on the assumption that, when applied at a sufficient concentration for an adequate duration, a toxicant exerts a selection pressure towards a more tolerant community. Assessment of structural and physiological parameters, the latter obtained by exposing a community to increasing amounts ot toxicant, not only demonstrates the effects of exposure, but also allows comparison of the level of toxicant contamination between various sites from which algal communities have been collected. Classical approaches, microscopy and incorporation of ${ }^{14} \mathrm{C}$, have already been applied to microalgal cummunities in complex experimental systems and in the field. However, environmental conditions may be expected to influence community selection by the toxicant and may hamper the validation of PICT in situ. Development of new methodologies to characterise community structure and tolerance is fundamental for advancement of knowledge of ecological risk assessment.

1.INRA, Laboratoire Toxicologie Environnementale, UMR INRA-UAPV Ecologie des Invertébrés, Agroparc, 84914 Avignon Cedex 9, France 2.INRA, Station d'Hydrobiologie Lacustre, 75 av. de Corzent, B.P. 511, 74203 Thonon-les-bains, France

3.INRA, Laboratoire d'Ecologie Aquatique, 65, rue de Saint Brieuc, 35042 Rennes Cedex, France

4.Corresponding author : berard@avignon.inra.fr 


\section{Introduction}

Les écosystèmes aquatiques sont soumis à des perturbations anthropiques de plus en plus fortes, qui résultent, entre autres, en une augmentation de la fréquence et du niveau de contamination par les toxiques. Dans ces écosystèmes, les algues sont des « cibles désignées « de ces contaminants, aussi bien par leur sensibilité intrinsèque (par exemple similitude forte au niveau physiologique avec les plantes terrestres visées par les traitements herbicides) que par leur rôle de support du réseau trophique aquatique (statut de « producteurs primaires «). En perturbant la physiologie des algues les plus sensibles et en diminuant ainsi leurs capacités compétitives, ces toxiques pourraient exercer une pression de sélection au sein des communautés algales au profit des espèces ou des clones les plus tolérants sans qu'aucun effet létal direct n'intervienne ou ne soit détectable (Bérard \& Pelte 1999). Il est donc possible, en conséquence, que certains polluants déstructurent les communautés algales des milieux contaminés et qu'ils provoquent éventuellement de ce fait, un déséquilibre de l'écosystème via le compartiment algal. Parmi les conséquences attendues de ces déséquilibres figurent des phénomènes de développement de fleurs d'eau - y compris celles impliquant des cyanobactéries toxiques -, de sédimentations importantes de matière organique d'origine phytoplanctonique, de désoxygénation et de déstructuration des niveaux supérieurs des réseaux trophiques...

Les études toxicologiques développées jusqu'à présent, impliquent généralement des organismes peu représentatifs des milieux naturels et des expérimentations en systèmes très simplifiés, ayant une signification écologique faible (Seguin et al. 2001). Par ailleurs, la composante écologique de l'écotoxicologie nécessite d'être développée afin de proposer des choix relatifs à la protection des écosystèmes (Chapman 2001). D'après Calow (1996), protéger la structure d'une communauté permettrait en général de protéger sa ou ses fonctions. Il est donc nécessaire de proposer et de valider des méthodes d'évaluation de la santé des écosystèmes basées à la fois sur l'étude des réponses physiologiques et sur la structure des communautés. Parmi ces communautés, les communautés algales sont particulièrement intéressantes à étudier car elles sont considérées comme de bons indicateurs de l'état des systèmes aquatiques et elles se situent à la base des réseaux trophiques, ce qui justifie leur étude d'un point de vue écotoxicologique (Mc Cormick \& Cairns 1994).

Luoma (1977) a suggéré que la tolérance élevée d'une population vis-à-vis d'un toxique précis indique que cette substance a exercé une pression de sélection sur cette population. Blanck et al. (1988) sont allés plus loin en appliquant cette théorie à une communauté entière et en proposant la méthode PICT (PollutionInduced Community Tolerance), comme outil de bioévaluation basé sur l'étude des conséquences d'une pollution sur la structure et la tolérance d'une communauté biologique.

\section{Principe et application du PICT}

\subsection{Objectifs et concept}

\section{- Objectifs de la méthode}

- Révéler une pollution toxique et ses effets sur les communautés biologiques dans un milieu naturel, en se basant sur l'hypothèse que la communauté préalablement exposée au toxique soupçonné présentera une tolérance accrue à ce toxique.

- Procurer au gestionnaire une information écotoxicologique complémentaire aux mesures biologiques qui caractérisent un écosystème (indices trophiques, par exemple) en détectant une pollution par un toxique, distincte d'un phénomène d'eutrophisation : en associant un test physiologique appliqué à la communauté échantillonnée exposée artificiellement au toxique incriminé, la méthode PICT permettrait d'associer la structure de la communauté au stress physiologique dû au toxique.

- Concept de base de la méthode : un toxique est susceptible d'exercer une pression de sélection au sein des communautés naturelles contaminées

La méthode PICT est fondée sur le fait qu'une communauté naturelle regroupe différents «composants» ayant une sensibilité différente vis-à-vis du polluant étudié. Ces «composants» peuvent être non seulement des espèces mais aussi des génotypes ou des phénotypes. Ainsi, les organismes les plus sensibles, exposés au toxique à une concentration et pendant une durée suffisante, ne sont plus concurrentiels et sont remplacés par des organismes plus tolérants. La structure des peuplements est alors modifiée : la communauté présente une tolérance supérieure vis-à-vis du polluant ou du type de polluant étudié, en comparaison à une communauté semblable mais n'ayant pas été exposée à une pression de sélection par le toxique. Cette différence de tolérance donne une indication sur l'état de pollution des milieux dans lesquels ont été prélevées les communautés.

Dans la pratique, la méthode PICT consiste à associer la mesure d'un descripteur de la structure (taxono- 
mie) de la communauté étudiée avec les résultats d'un test écophysiologique global et rapide (en réalisant une mesure d'activité, par exemple) appliqué à cette communauté lorsqu'on l'expose en conditions contrôlées à des concentrations croissantes du toxique étudié (Fig. 1). Les résultats obtenus (courbes «concentration-réponse», Concentrations Efficaces ...), associés aux données taxonomiques, permettent d'apprécier la structure et la tolérance de ces communautés vis-à-vis du toxique étudié, et ainsi de préciser et de comparer l'état de contamination par ce toxique des sites dans lesquels les communautés ont été échantillonnées.

\subsection{Application}

Dans une étude inter-Agences réalisée par Vindimian \& Garric (1993) visant à choisir des bio-indicateurs susceptibles d'indiquer le niveau de contamina-

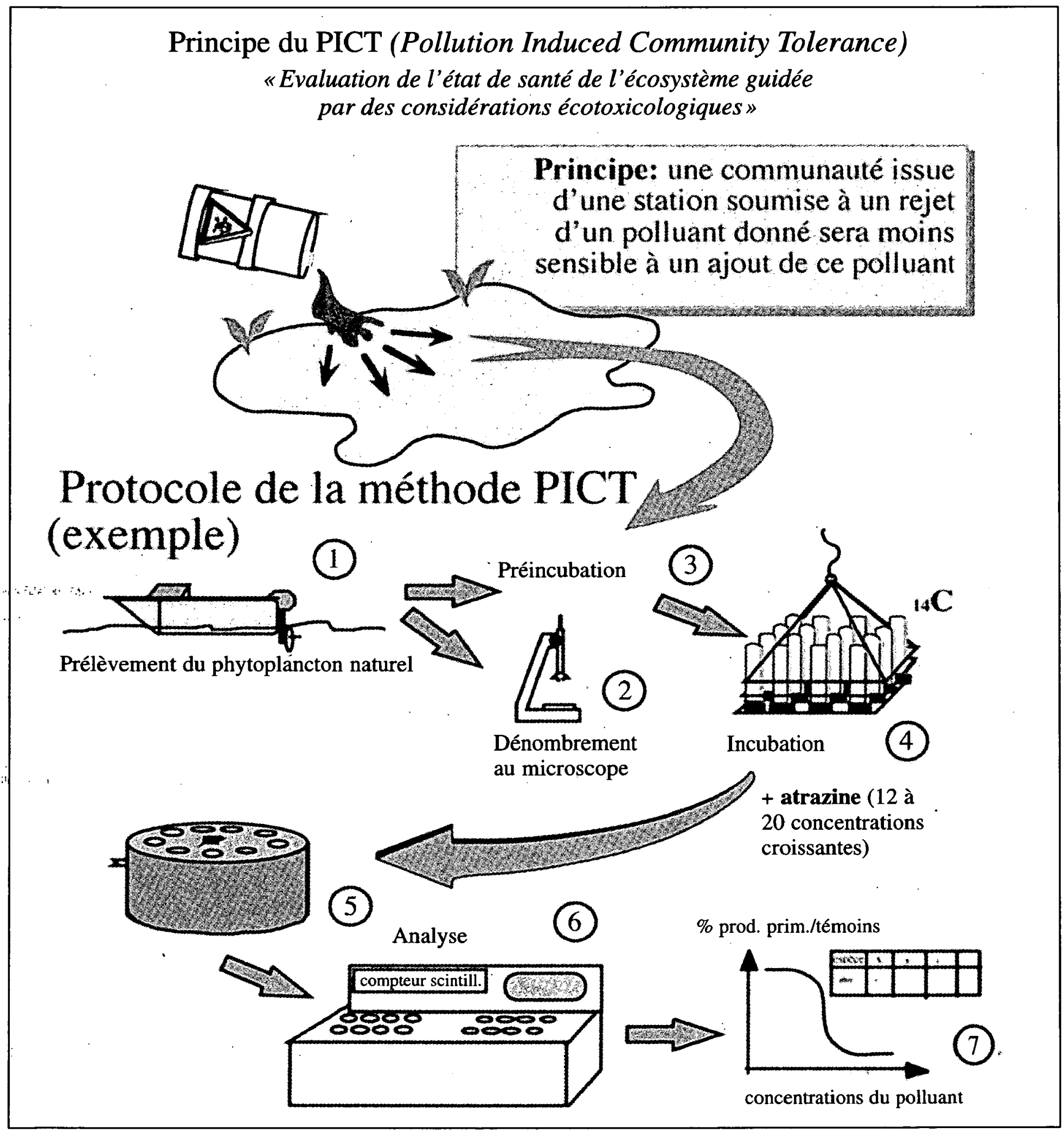

Fig. 1. Exemple de protocole pour l'application de la méthode PICT.

Fig. 1. An example of protocol to perform the PICT. 
tion d'un milieu, la méthode PICT était considérée comme un indicateur intéressant, ayant un degré de signification écologique important mais qui nécessitait des études scientifiques complémentaires.

Cette approche a été appliquée sur des communautés algales et microbiennes aquatiques (phytoplancton, périphyton, epipsammon : Admiraal et al. 1999 ; Dahl 1996 ; Dahl \& Blanck 1996b). Dans le principe, elle pourrait s'appliquer à n'importe quel type de communauté - elle a été utilisée pour mettre en évidence les impacts du cuivre sur des communautés de nématodes aquatiques (Millward \& Grant 1995) - à partir du moment où les questions d'échantillonnage sont résolues et les moyens d'évaluer la composition taxonomique et la tolérance de la communauté complète sont efficaces.

La méthode PICT est susceptible de s'appliquer aussi bien dans le cadre d'études d'évaluation de la qualité des milieux que dans le cadre d'études traitant de l'évaluation du risque écotoxicologique a priori en systèmes expérimentaux complexes.

Quelques études de ce type ont jusqu'à présent été réalisées en milieu naturel (Tableau 1). Elles concernent surtout les métaux ou leurs dérivés : TBT (Blanck \& Dahl 1996-2000), cuivre (Gustavson et al. 1999), arsenic (Wangberg 1995, Knauer et al. 2000), zinc (Admiraal et al. 1999), la plupart concernant des pollutions non diffuses et bien identifiées. Une étude in situ a permis d'évaluer la zone d'influence d'une source ponctuelle de pollution aérienne par l'arseniate, sur les communautés phytoplanctoniques de lacs situés autour de cette source de pollution (Wangberg 1995). Quatre études appliquant la méthode PICT ont été réalisées sur des systèmes naturels contaminés par des pesticides (Bérard et al. 1998, Guash et al. 1999, Nyström et al. 1999, Bérard et al. 2001c). La première a comparé la tolérance à l'atrazine de communautés phytoplanctoniques échantillonnées dans deux lacs différents et la deuxième a montré l'influence de la contamination par l'atrazine, mais aussi par d'autres paramètres comme les nutriments ou la lumière, sur la tolérance du périphyton à cet herbicide. La troisième étude met en évidence l'influence d'herbicides sulfonylurées sur la tolérance du périphyton de deux rivières ; ces herbicides n'agissent pas directement sur la photosynthèse et les tests physiologiques employés sont basés sur des activités non spécifiques aux algues (e.g. incorporation de l'adénine tritiée). Enfin, la dernière étude a comporté plusieurs campagnes réalisées sur une rivière en zone d'agriculture intensive. Elle a mis en évidence d'une part, l'intérêt d'utiliser en association le phytoplancton et le périphyton selon le faciès (lentique ou lotique) de la rivière comme indicateurs de pollutions, et d'autre part, le caractère intégrateur de ces communautés algales prélevées dans leur milieu.

Outre les difficultés rencontrées par les auteurs pour mettre en évidence l'acquisition de tolérance induite par un herbicide inhibiteur du Photosystème II (PS II : Gustavson \& Wängberg 1995, Dahl \& Blanck 1996 ; Nyström et al. 2000, Bérard \& Benninghoff 2001), les études in situ concernant les pollutions diffuses d'origine agricole sont d'autant plus difficiles à mettre en œuvre que ces milieux sont sujets aux variabilités d'apports en ces polluants et aux effets de dilution associés aux différents régimes dans les rivières (Jurgensen \& Hoagland 1990, Lakshminarayana et al. 1992).

Dans le cadre des études visant à l'évaluation des risques écotoxicologiques liés à la contamination de l'environnement par les xénobiotiques et les métaux (homologation des pesticides, autorisations de rejets pour des industriels ...), les décideurs exigent de plus en plus souvent de disposer d'informations obtenues à partir d'expérimentations en systèmes complexes comme les microcosmes et les mésocosmes aquatiques (Pascoe et al. 2000). Une fois ces systèmes mis en place, la question se pose de savoir quels sont les paramètres révélateurs du risque qui doivent être mesurés ? Afin de valoriser au maximum les propriétés des outils de type mésocosme ou microcosme, il est nécessaire de choisir des paramètres de bio-évaluation pertinents d'un point de vue écologique, simples d'utilisation et cractéristiques d'une communauté (voire de la dynamique de cette communauté) plutùt que des individus. La méthode PICT répond tout à fait à ces critères. Jusqu'à présent les différentes études appliquant cette méthode en systèmes expérimentaux n'avaient pas pour objectif premier l'évaluation du risque, mais plutUt la validation de cette méthode appliquée à différentes communautés avec des toxiques variés (Tableau 1) en conditions en partie contrôlées. Récemment, une étude sur du phytoplancton de mésocosmes aquatiques lentiques contaminés par de l'atrazine (Seguin et al. 2000) a été réalisée dans une démarche comparable à celle employée pour l'évaluation du risque a priori. Les auteurs ont montré expérimentalement que la tolérance à l'atrazine, très rapidement acquise, fluctuait au cours de du temps (probablement du fait de phénomènes associés aux successions algales et aux fluctuations des caractéristiques du milieu dans les mésocosmes), mais qu'elle restait toujours plus élevée dans les systèmes contaminés que dans les mésocosmes témoins. Ces résultats montrent que la méthode PICT est un outil efficace dans le cadre de ce type d'évaluation et pour cette molécule. 


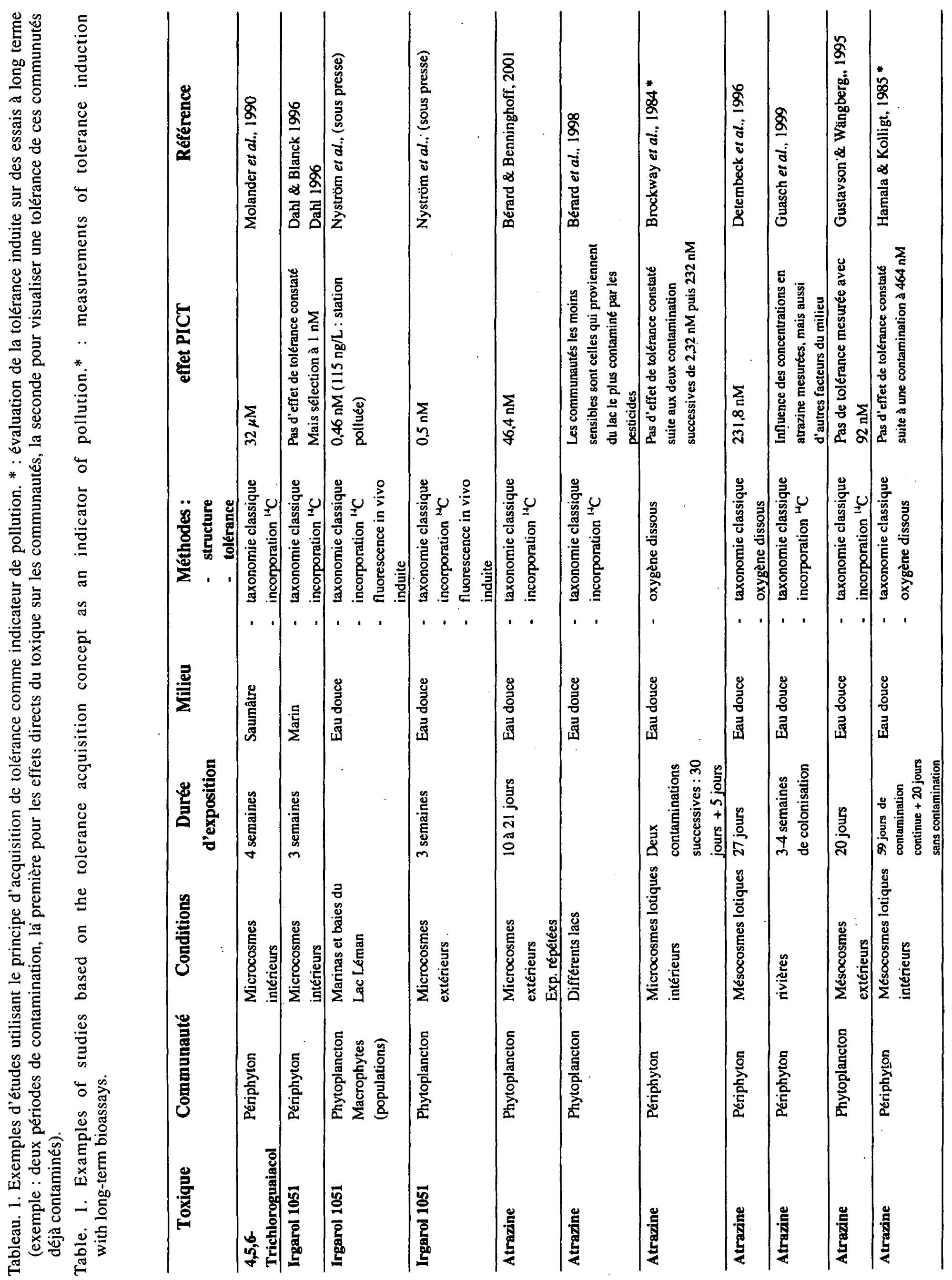




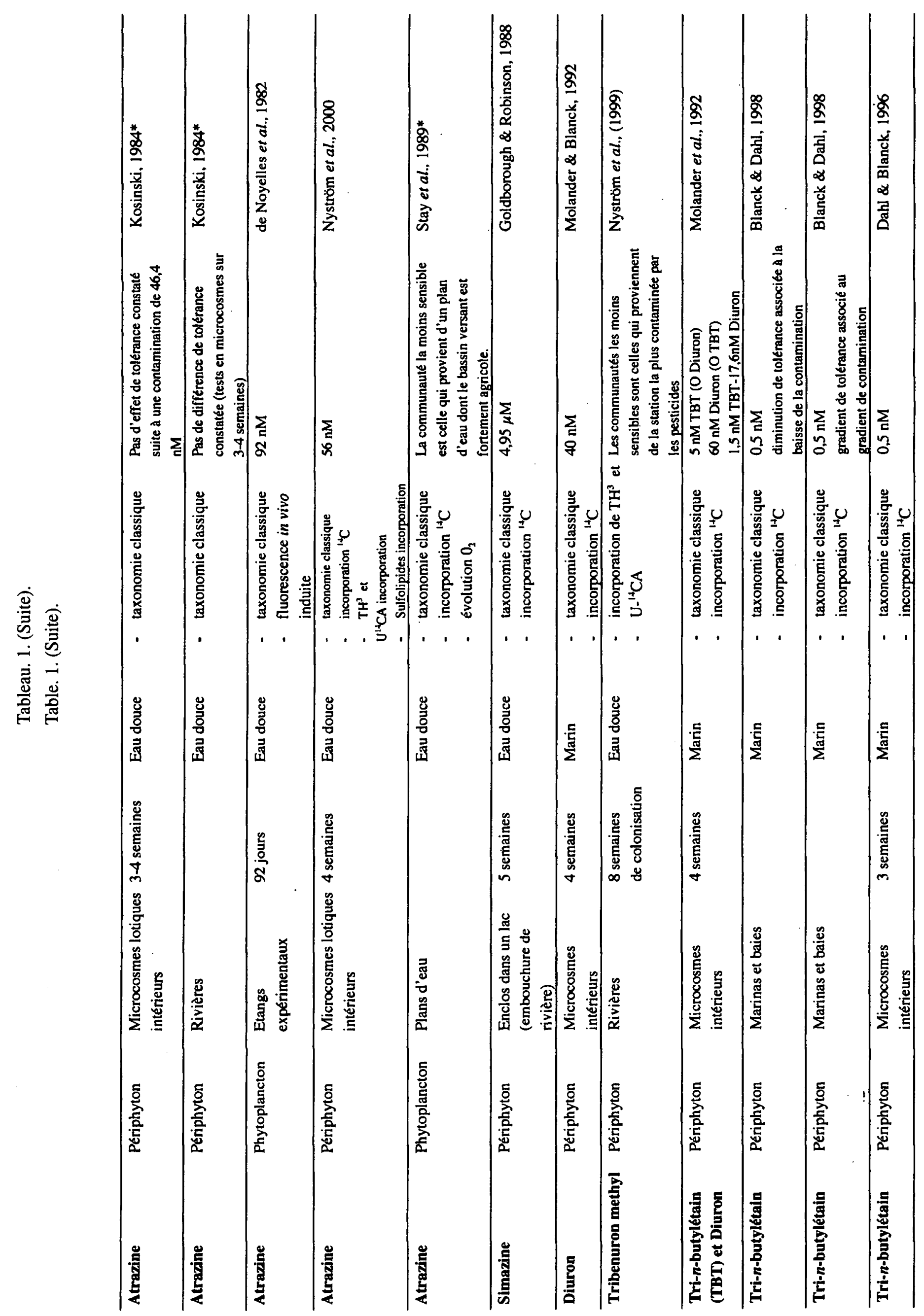




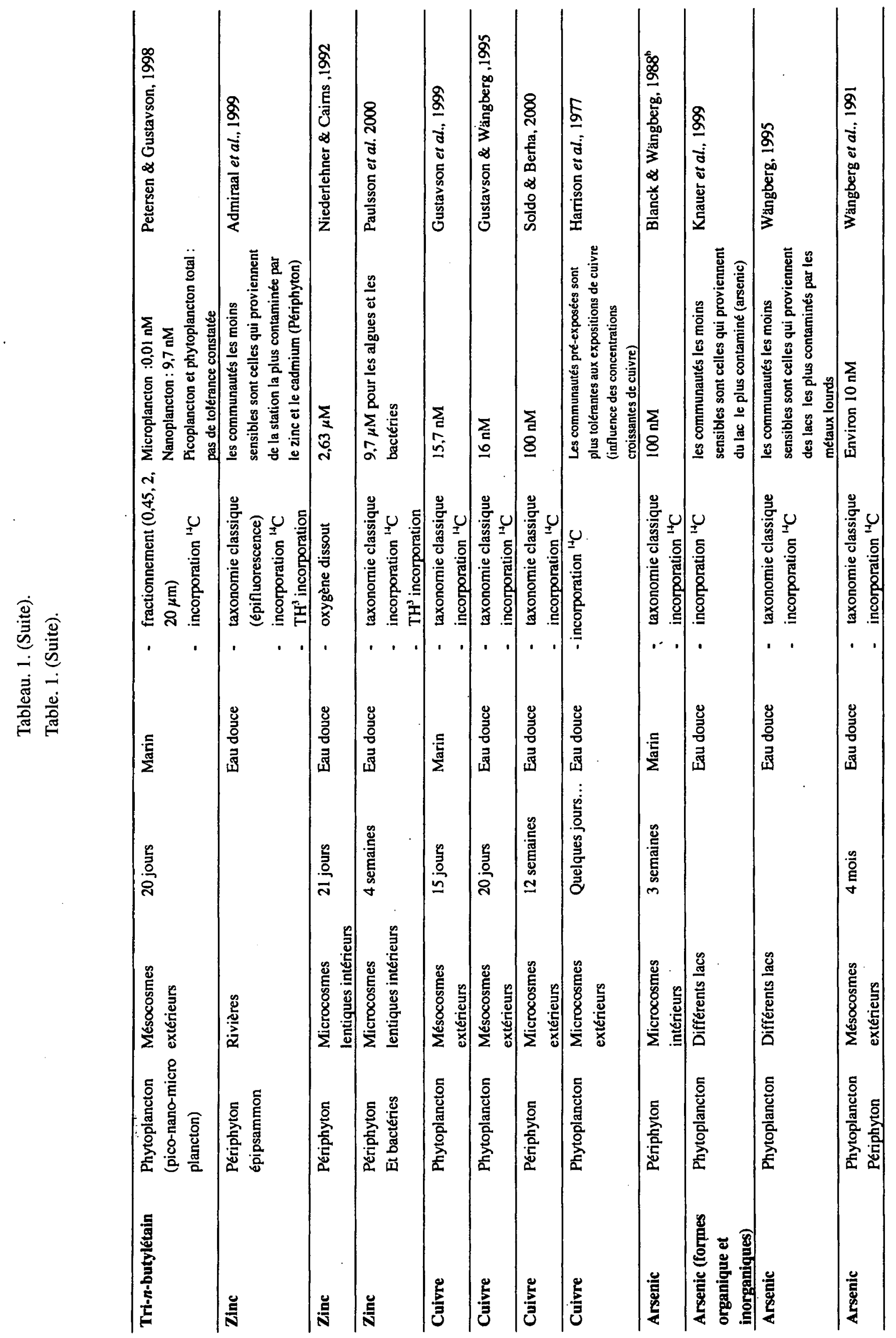




\section{Processus de sélection : mise en évidence de la tolérance et validation de la méthode}

\subsection{Processus de sélection au sein de la communau- té \\ La méthode PICT reflète l'existence de deux niveaux de sélection.}

Le premier concerne le niveau intraspécifique : la sélection par le toxique au sein d'une population peut induire une augmentation de tolérance dans la population concernée. Il existe de nombreux exemples mettant en évidence des phénomènes de sélection de populations d'algues en milieux naturels plus ou moins contaminés par des xénobiotiques ou des métaux (Foster 1982, Cosper et al. 1984, Hersh \& Crumpton 1989, Takamura et al. 1989, Kasai et al. 1993, Bérard et al. 1998).

Le second niveau de sélection est interspécifique. De nombreuses études ont montré qu'un toxique était susceptible de sélectionner certaines espèces au détriment des autres au sein d'une communauté algale (e.g. Ruth 1978, Bryfogle \& Mc Diffet 1979, Pérès et al. 1997, Bérard \& Pelte 1999, DeLorenzo et al. 1999). Les travaux de Faber et al. (1998), utilisant un compteur à cytométrie de flux associé à un fluorimètre (mesures de fluorescence in vivo indiquant l'état de viabilité de chaque cellule algale), ont mis en évidence les impacts de deux herbicides (glufosinate-ammonium et bialaphos) sur la structure du phytoplancton lacustre dans des enceintes. Loez et al. (1995) ont montré les effets sélectifs du zinc sur des communautés phytoplanctoniques provenant de rivières et cultivées au laboratoire : selon la concentration de zinc, différents groupes algaux étaient inhibés ou stimulés. Payne \& Price (1999) ont montré que la variabilité importante de sensibilité vis-à-vis du cadmium, mise en évidence sur différentes espèces phytoplanctoniques marines, était associée à des différences de capacité de détoxication entre ces espèces. Kasai (1999) a montré sur différentes espèces et groupes d'espèces du périphyton, isolés à partir de sites pollués par des herbicides, que, selon le groupe d'algues (chlorophycées ou diatomées), la gamme de sensibilité à l'herbicide, et donc le potentiel de sélection par l'herbicide, étaient variables. La même équipe (Takamura et al. 1990) a montré au moyen d'essais réalisés sur 11 espèces de cyanobactéries et 7 espèces de microalgues eucaryotes, que les cyanobactéries présentaient des capacités d'adaptation vis-à-vis du cuivre beaucoup plus élevées. Ces différences de potentiel adaptatif des espèces, vis-à-vis d'un toxique, participent probablement à la diversité des réponses au stress, des potentiels de sélection et d'acquisition de tolérance des communautés algales.

D'après de Noyelles et al. (1982), les interactions interspécifiques peuvent influencer la réponse de la communauté planctonique vis-à-vis du toxique, car le phénomène de sélection des espèces de microphytes par le jeu des relations interspécifiques modifie la réaction de chaque espèce au toxique. Les interactions interspécifiques sont particulièrement complexes, avec des compétitions directes ou indirectes (voir Tilman 1977, Sommer 1986), mais probablement aussi avec des phénomènes de synergie ou d'inhibition (interactions allélopathiques : Wolfe \& Rice 1979, Mayasich et al., 1987).

La sélection peut varier selon les paramètres du milieu en interaction avec le toxique. Différentes études sur les métaux ont montré l'influence des caractéristiques du milieu (variable selon les sites étudiés et les saisons mais aussi selon les communautés présentes) sur la biodisponibilité du toxique et donc sa toxicité (voir Slauenwhite et al. 1991, Taub et al. 1991, Winner \& Owen 1991). Les facteurs du milieu peuvent aussi influencer directement la sensibilité des algues au toxique et influencer la sélection des communautés algales : plusieurs études avec des herbicides ont démontré l'influence de la lumière, des concentrations en nutriments et de la température de l'eau sur la sensibilité et la sélection de populations et de communautés algales (voir Lampert et al. 1989, Millie et al. 1992, Caux \& Kent 1995, Guasch \& Sabater 1998, Bérard et al. 1999b). La biomasse, l'état physiologique et la structure de la population ou de la communauté stressée, peuvent influencer sa sensibilité et sa sélection par le toxique (voir par exemple Ramirez Torres \& O'Flaherty 1976, Herman et al. 1986, Mayasich et al. 1987, Bérard et al. 1999a). Les conditions du milieu sont donc susceptibles d'influer sur la pression de sélection au sein de la communauté et, de ce fait, sur l'acquisition de tolérance.

Les méthodes d'évaluation de la sélection ont été jusqu'à présent basées principalement sur l'analyse taxonomique classique à l'aide d'un microscope inversé pour le phytoplancton et le périphyton et à l'aide d'un microscope à épifluorescence pour le périphyton et les algues des sédiments. Des méthodes nouvelles, basées sur la chémotaxonomie et la biologie moléculaire sont actuellement mises en ouvre.

Les approches basées sur l'utilisation des techniques de biologie moléculaire peuvent permettre d'évaluer la diversité intraspécifique mais aussi la diversité interspécifique. En fonction des séquences-cibles étudiées, il est en effet possible de choisir des marqueurs molé- 
culaires plus ou moins polymorphes et donc susceptibles d'apporter des informations à différents niveaux (espèce, genre...). C'est ainsi qu'en s'intéressant à l'ADN ribosomal $18 \mathrm{~S}$, il est possible d'obtenir des informations sur la diversité interspécifique d'une communauté, alors que l'étude d'une séquence plus variable telle que l'ITS1 (Internal Transcribed Spacer 1, entre l'ADN $18 S$ et l'ADN 23S) peut permettre d'obtenir des informations sur la diversité intraspécifique.

Cette approche a été récemment testée sur des communautés périphytiques. Pour chaque site étudié, un fragment d'ADN $18 \mathrm{~S}$ a été amplifié à partir d'ADN extrait sur l'ensemble de la communauté. Les fragments amplifiés chez les diverses espèces présentes ont été ensuite clonés. Le criblage de la banque d'ADN $18 \mathrm{~S}$ ainsi obtenue a été réalisé par séquençage aléatoire (entre 10 et 15 clones par échantillon). La diversité de chaque station a ainsi pu être évaluée sur tous les groupes taxonomiques présents (champignons, algues, etc : Dorigo 2001). D'autres techniques moléculaires telles que la DGGE (Denatured Gel Gradient Electrophorese) ou la SSCP (Single Strand Conformation Polymorphism) sont en cours de développement (voir Murray et al. 1996, Engelen et al. 1998, Mc Caig et al. 1999, Dahllöf et al. 2000, Fantroussi et al. 1999); elles pourraient être adaptées aux communautés algales eucaryotes. Elles permettent une comparaison très rapide de la diversité spécifique de communautés distinctes.

\subsection{Mise en évidence de la tolérance de la commu- nauté}

La mise en œuvre de la méthode PICT nécessite la comparaison du niveau de tolérance de différentes communautés vis-à-vis d'un même toxique. Ces mesures de tolérance, réalisées au moyen de tests physiologiques globaux, sont donc relatives : la tolérance d'une communauté est évaluée en comparaison à la tolérance d'une communauté, a priori, non exposée au toxique, et considérée comme référence. La question est donc de définir cette référence.

Une communauté de référence doit être une communauté qui est susceptible d'acquérir une tolérance importante après avoir été exposée au toxique étudié. Cette acquisition de tolérance dépend non seulement de la communauté elle-même mais aussi de ses interactions avec le toxique. Une communauté présentant initialement une diversité d'espèces et de clones aux sensibilités très différentes vis-à-vis du toxique aura a priori un potentiel de sélection, donc d'acquisition de tolérance, important (Blanck et al. 1988).

Dans certains cas, l'acquisition de tolérance est difficile à détecter et des acquisitions de sensibilité sont parfois constatées. Ainsi, Nyström et al. (2000) ont mis en évidence pour du périphyton, une acquisition de tolérance faible pour une exposition chronique à des concentrations en atrazine comprises entre 0,056 et $0,56 \mu \mathrm{M}$ dans des microcosmes, suivie d'une diminution de tolérance pour des concentrations en atrazine plus élevées. En conditions expérimentales (microcosmes extérieurs, phytoplancton d'eau douce) une diminution de tolérance des communautés exposées à un fongicide (prochloraze), a été observée (Bérard et al. 2001a). L'hypothèse proposée est que le prochloraze, inhibiteur de différentes activités métaboliques, inhiberait directement les activités de détoxication des algues, les rendant encore plus sensibles au fongicide lui-même.

Dans le cas d'études in situ, il faut prendre en compte l'influence des paramètres du milieu qui sont susceptibles d'interagir avec le toxique et les algues. Des interactions entre le toxique, les paramètres du milieu et les communautés algales peuvent avoir des conséquences sur les phénomènes de sélection (voir plus haut) et la tolérance induite peut donc être indirectement influencée (Bérard \& Benninghoff 2001).

La tolérance induite dépend aussi de la sensibilité initiale de la communauté («baseline tolerance», Molander 1991). Cette sensibilité initiale dépend aussi des conditions du milieu et des caractéristiques (biomasse, niveau d'activité, structure) de cette communauté. Par exemple, Guasch \& Sabater (1998) ont montré sur du périphyton de rivière que celui-ci présentait une sensibilité variable à l'atrazine selon les conditions régnant lors de la colonisation (en particulier la lumière) et sa composition (espèces de diatomées présentes). $\mathrm{Au}$ cours d'une série d'expérimentations en microcosmes extérieurs, il a été montré que le phytoplancton présentait une sensibilité à l'atrazine qui variait selon la température, la lumière, la concentration initiale en orthophosphates et la diversité initiale de l'inoculum cultivé (Bérard \& Benninghoff 2001). Nyström et al. (1999) ont constaté en rivière une variabilité de la tolérance pour des communautés périphytiques vis-à-vis du tribenuron-méthyl, un herbicide de la famille des sulfonylurées, dans une station de référence. Sachant que les concentrations en acides aminés dans le milieu sont susceptibles d'influer sur la sensibilité des algues aux sulfonylurées (herbicides inhibiteurs de la synthèse de certains acides aminés), ces auteurs émettent l'hypothèse que les variations saisonnières de concentrations en acides aminés dans le milieu impliqueraient des variations saisonnières de sensibilité des communautés algales aux sulfonylurées. Les travaux de Wangberg sur le périphyton et le phytoplancton de systèmes 
aquatiques variés ont montré l'influence du phosphore sur la sensibilité et l'acquisition de tolérance des algues vis-à-vis de l'arsenic (Blanck \& Wängberg 1988a, Wängberg \& Blanck 1990, Wängberg et al. 1991). Une étude récente de Petersen \& Gustavson (1998) a mis en évidence des différences de tolérance induite par le TBT en rapport avec la taille des algues phytoplanctoniques testées. L'hypothèse émise par ces auteurs est que, selon la taille des organismes, ceux-ci sont soumis à une pression de broutage et à des compétitions pour les nutriments différentes, ce qui modifierait leur sensibilité initiale au TBT et en conséquence leur tolérance. Cette différence de sensibilité à court terme des communautés algales, selon la taille des organismes, a déjà été mise en évidence dans les travaux de Munawar et al. (1987). Ces résultats confirment bien l'importance des interactions interspécifiques dans la sensibilité et l'acquisition de tolérance des communautés aux toxiques.

Le choix du test physiologique pour évaluer la tolérance de la communauté est fondamental car sa réponse dépend d'une part du mode d'action du toxique, et d'autre part des mécanismes de tolérance de la communauté (Molander 1991). Ainsi, Nyström (1997) a testé différentes mesures d'activité (incorporation du $\mathrm{C}^{14}$, de l'adénine, de la leucine et de la thymidine tritiée, incorporation des sulfolipides) sur du périphyton exposé à de l'atrazine ou du zinc : l'incorporation de l'adénine s'est avérée être plus sensible vis-à-vis du zinc, alors que cette mesure d'activité globale était peu sensible vis-à-vis de l'atrazine.

L'association d'outils de mesures variés d'activités (activités hétérotrophes et autotrophes) permet de mettre en évidence d'éventuelles interactions entre différentes communautés. Nyström (1997) a ainsi mis en évidence un remplacement des communautés autotrophes par des communautés hétérotrophes dans les microcosmes contaminés par l'atrazine.

D'autres méthodes d'évaluation de la tolérance sont actuellement mises en œuvre. Par exemple, l'induction de fluorescence utilisée par de Noyelles et al. (1982) est développée dans notre laboratoire et comparée à l'incorporation du C14, pour mettre en évidence l'apparition de tolérance provoquée par des inhibiteurs de la photosynthèse (Dorigo \& Leboulanger 2001, Bérard et al. 2001b, Seguin et al. 2002, Nyström et al. 2002). Les avantages de cette méthode, basée sur la fluorescence in vivo des algues (phytoplancton et périphyton), sont qu'elle n'utilise pas de radio-éléments, que les résultats sont obtenus en même temps que ceux du test et que, dans le cas d'une contamination par des inhibiteurs du PS II, cette méthode est très sensible.

\subsection{Validation de la méthode PICT}

La plupart des études expérimentales réalisées sur la méthode PICT comparent des paramètres classiques de mesure du stress à long terme (Tableau 2) tels que la biomasse et la structure (concentration en chlorophylle, poids sec, dénombrements totaux, indices de diversité, ...), et la production primaire (incorporation du $\mathrm{C}^{14}$, mesures d'oxygène dissous ...). La méthode PICT est alors généralement bien validée, les résultats de tolérance acquise sont en accord avec les mesures de biomasse ou d'activité réalisées au cours de l'expérimentation. Cependant, il existe certains cas où les méthodes classiques de mesure du stress à long terme semblent plus sensibles que celle mise en œuvre dans l'approche PICT. Par exemple, Paulsson et al. (2000) ont constaté une acquisition de tolérance au zinc de communautés périphytiques à des concentrations plus élevées que celles entrainant une différence de structure entre microcosmes témoins et microcosmes contaminés. L'hypothèse proposée est que le zinc interagirait avec le phosphore, provoquant une limitation des algues vis-à-vis du phosphore. Cet effet indirect du zinc sur les algues semble donc plus important que l'effet direct sur leur acquisition de tolérance. Les résultats concernant les inhibiteurs du PS II mènent aussi à des conclusions. En particulier, Gustavson \& Wängberg (1995) et Molander \& Blanck (1992) ne valident pas la méthode avec l'atrazine et le diuron, mais la majorité des études expérimentales valident la méthode à des concentrations variables de ces herbicides (Tableau 1). Comme nous l'avons déjà présenté, le rôle des paramètres du milieu sur ces acquisitions de tolérance semble important et, comme toute étude écologique, une seule campagne ou une seule expérimentation n'est probablement pas suffisante pour mettre en évidence un phénomène d'acquisition de tolérance sur des communautés naturelles soumises aux variations saisonnières (Bérard \& Benninghoff 2001, Bérard et al. 2001a).

Une autre question se pose à propos de la validation de la méthode : certains auteurs ont mis en évidence des phénomènes de co-tolérance entre toxiques (Tableau 2). La co-tolérance apparaît quand une population ou une communauté exposée à une pression de sélection par un toxique, acquiert une tolérance accrue par rapport à ce premier toxique, mais aussi par rapport à d'autres toxiques. Ce phénomène de co-tolérance est susceptible de se produire lorsque les toxiques présentent une structure chimique ou un mode d'action comparables. Les mécanismes impliqués sont des phénomènes de détoxication communs (métabolisation, chélation des métaux lourds par exemple ; Soldo \& Behra 

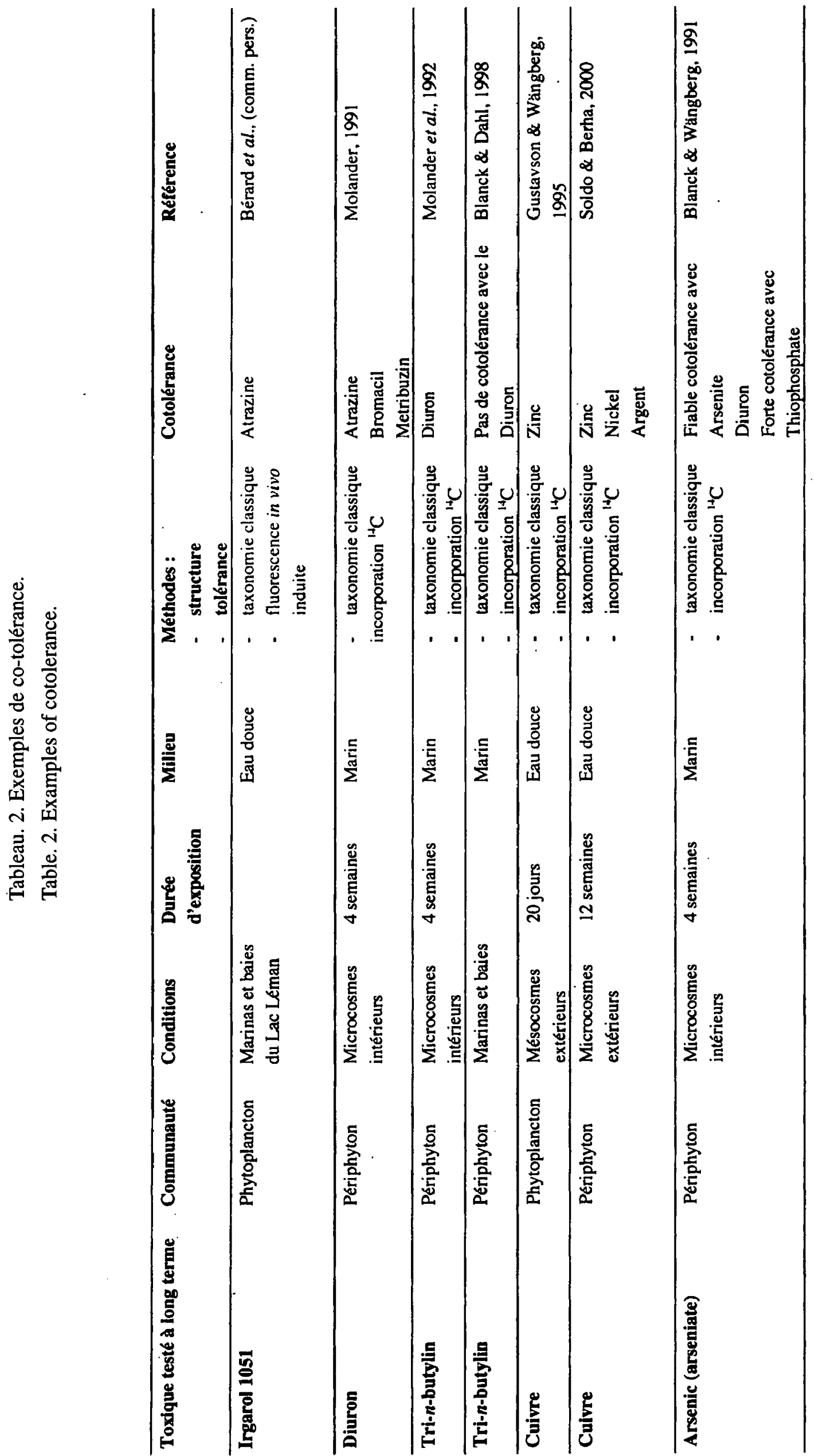
2000) ou des modifications d'une cible moléculaire commune (cas des inhibiteurs du PS II : Blanck et al. 1988). Une combinaison de ces différents mécanismes peut se produire et compliquer l'interprétation des phénomènes observés (Molander 1991).

La co-tolérance est donc un phénomène avéré, à ne pas confondre avec la «multiple tolérance» de la communauté soumise à plusieurs toxiques. S'il est alors difficile de séparer l'effet PICT de deux toxiques proches, la méthode PICT permet de séparer des groupes de molécules ayant des effets communs, que l'on peut associer à des types de pollutions (herbicides inhibiteurs du PS II, groupes de métaux lourds, Tableau 1). Toutefois, les études de co-tolérance sont encore assez peu nombreuses et axées sur les effets à long terme de quelques molécules seulement.

\section{Conclusion}

La méthode PICT est un outil d'évaluation écotoxicologique ayant une signification écologique importante puisqu'elle intègre la variabilité de la communauté entière avec les phénomènes d'adaptation physiologique des organismes et de sélections intra et inter-spécifique. Peu d'outils de bioévaluation des milieux naturels soumis à des contaminations toxiques sont à notre disposition, cette méthode mérite donc d'être développée pour participer à l'évaluation des risques écotoxicologiques dans l'environnement.

Toutefois, cette revue bibliographique montre que la méthode n'a encore été validée qu'avec peu de molécules et qu'elle a été peu appliquée in situ. De plus, son utilisation est parfois difficile selon les conditions du milieu, la nature des tests physiologiques et les molécules testées. Il est donc nécessaire de développer les études traitant des interactions entre toxiques et paramètres environnementaux susceptibles d'aggraver ou de limiter les conséquences d'une pollution détectée par la méthode PICT (Nyström 1997). Plus précisément, des travaux appliquant le PICT à des communautés variées (microorganismes hétérotrophes, autotrophes, invertébrés ...) et dans des environnements variés (eau libre, biofilms, sédiments, sols : Saint Olive et al. 2001), permettraient de valider la méthode et de préciser certains points quant à sa mise en oeuvre. Enfin, le développement de méthodologies nouvelles pour caractériser la structure et la tolérance des communautés testées est fondamental pour progresser dans nos connaissances sur le risque écotoxicologique.

\section{Remerciements}

Nous remercions l'Agence de l'Eau Loire-Bretagne (sous-direction Prospectives, Xavier Bourrain) qui a participé au financement de cette étude bibliographique.

\section{Travaux cités}

Admiraal W., Blanck H., Buckert-de Jong M., Guasch H., Ivorra N., Lehmann V., Nyström B.A.H., Paulsson M. \& Sabater S. 1999. Short-term toxicity of zinc to microbenthic algae and bacteria in a metal polluted stream. Water Res., 33 : 1989-1996.

Bérard A. \& Benninghoff C. 2001. - Pollution-Induced Community Tolerance (PICT) and seasonal variations in the sensitivity of phytoplankton to atrazine in nanocosms. Chemosphere, $45: 427$ 437.

Bérard A., Bosse J.P., Cauzzi N., Dorigo U., Druart J.C., Humbert J.F., Leboulanger C., Nyström B., Saint-Olive A. \& Seguin F. 2001a. - La méthode PICT (Pollution-Induced Community Tolerance) appliquée aux communautés algales. Faisabilité et application. Rapport INRA Thonon - Agence de l'Eau Loire-Bretagne, SHL199-2001: $46 \mathrm{p}$.

Bérard A., Dorigo U., Seguin F. \& Leboulanger C. 2001b. - Risk assessment and diagnostic of pollution : use of chlorophyll fluorescence as endpoint in revealing PICT in algal communities contaminated by PSII inhibitors. SETAC Europe $11^{\text {th }}$ Annual Meeting. Madrid 6-10 mai 2001. Abstract $n^{\circ}$ W/MF161.

Bérard A., Dorigo U., Humbert J.F., Bourrain X., Cauzzi N., Druart J.C. \& Leboulanger C. 2001c. - Révélation d'une pollution diffuse par la tolérance induite chez les communautés de microalgues. Application de la méthode PICT (Pollution-Induced Community Tolerance) dans une rivière contaminée par des herbicides. C.R. XXXIème Congrès du Groupe français des Pesticides, Lyon : 15-17 mai $2001: 79$.

Bérard A. \& Pelte T. 1999. - Les herbicides inhibiteurs du photosystème II (PSII), effets sur les communautés algales et leur dynamique. Rev. Sci. Eau, 12: 333-361.

Bérard A., Pelte T. \& Druart J.C. 1999a. - Seasonal variability in the sensitivity to atrazine of lake Geneva phytoplankton community structure. Arch. Hydrobiol., $145: 277-295$.

Bérard A., Leboulanger C. \& Pelte T. 1999b. - Seasonal and temperature dependence of Oscillatoria limnetica Lemmermann tolerance to atrazine among natural phytoplankton populations and in pure culture. Arch. Environ. Contam. Toxicol., 37 : 472-479.

Bérard A., Pelte T., Menthon E., Druart J.C. \& Bourrain X. 1998. Caractérisation du phytoplancton de deux systèmes limniques vis-à-vis d'un herbicide inhibiteur de la photosynthèse : la méthode PICT, application et signification. Ann. Limnol. 34 : 269-282.

Blanck H. \& Dahl B. 1996. - Pollution-induced community tolerance (PICT) in marine periphyton in a gradient of tri-n-butyltin (TBT) contamination. Aquat. Toxicol., $35: 59-77$.

Blanck H. \& Dahl B., sous presse. - Recovery of marine periphyton communities around a Swedish marina after the ban of TBT use in antifouling paint. Mar. Poll. Bull.

Blanck H. \& Wängberg S.-A. 1988a. - Validity of an ecotoxicological test system : Short-Term and long-term effects of arsenate on marine periphyton communities in laboratory systems. Can. J. Fish. Aquat. Sci., 45 : 1807-1815.

Blanck H. \& Wängberg S.-A. 1988b. - Induced community tolerance in marine periphyton established under arsenate stress. Can. J. Fish. Aquat. Sci., 45 : 1816-1819.

Blanck H. \& Wängberg S.-A. 1991. - Pattern of cotolerance in marine periphyton communities established under arsenate stress. Aquatic Toxicol., $21: 1-14$. 
Blanck H., Wängberg S.-A. \& Molander S. 1988. - Pollution-Induced Community Tolerance - A new ecotoxicological tool. In Cairns J. Jr. \& J.R. Pratt. (eds) Functional Testing of Aquatic Biota for Estimating Hazards of Chemicals. ASTM STP 1988. American Society for Testing and Materials, Philadelphia, 219-230.

Brockway D.L., Smith P.D \& Stancil F.E. 1984. - Fate and effects of atrazine in small aquatic microcosms. Bull. Environ. Contam. Toxicol., 32 : 345-353.

Bryfogle B.M \& McDiffett W.F. 1979. - Algal succession in laboratory microcosms as affected by an herbicide stress. Am. Midl. Nat., $101: 344-354$.

Chapman P.M. sous presse.- Integrating toxicology and ecology : putting the « eco « into ecotoxicology. Mar. Poll. Bull.

Calow P. 1996. - Ecology in ecotoxicology : some possible 'rules of thumb'. In : Baird, D.J., Maltby, L., Greig-Smith, P.W., Douben, P.E.T. (eds.), Ecotoxicology : Ecological Dimensions. Chapman and Hall, London : 5-12.

Caux P.-Y. \& Kent R.A. 1995.- Towards the development of a sitespecific water quality objective for atrazine in Yamaska River, Quebec, for the protection of aquatic life. Water Qual. J. Can., $30: 157-178$.

Cosper E.M., Wurster C.F. \& Rowland R.G. 1984. - PCB resistance within phytoplankton populations in polluted and unpolluted marine environments. Mar. Environ. Res., 12 : 209-223.

Dahl B. 1996. - On the ecotoxicology of antifouling agents. The use of marine microalgal communities in predictive and retrospective assessments. Thèse de Doctorat, Université de Göteborg, Suède, $45 \mathrm{pp}$.

Dahl B. \& Blanck H. 1995.- Pollution-induced community tolerance (PICT) in periphyton communities established under tri-nbutyltin (TBT) stress in marine microcosms. Aquat. Toxicol., 34 : 305-325.

Dahl B. \& Blanck H. 1996a.- Toxic effects of the antifouling agent Irgarol 1051 on periphyton communities in coastal water microcosms. Mar. Pollut. Bull., 32 : 342-350.

Dahl B. \& Blanck H. 1996b. - Use of sand-living microalgal communities (epipsammon) in ecotoxicological testing. Mar. Ecol. Prog. Ser., 144 : 163-173.

Dahllöf I., Baillie H. \& Kjelleberg S. 2000. — rpoB-based microbial community analysis avoids limitations inherent in $16 \mathrm{~S}$ rRNA gene intraspecies heterogeneity. Appl. Environ. Microbiol., 66 : 3376-3380.

DeLorenzo M.E., Lauth J., Pennington P.L., Scott G.I. \& Ross P.E. 1999. - Atrazine effects on the microbial food web in tidal creek mesocosms. Aquat. Toxicol., $46: 241-251$.

Detenbeck N.E., Hermanutz R., Allen, K. \& Swift C. 1996. - Fate and effects of the herbicide atrazine in flow-through wetland mesocosms. Environ. Toxicol. Chem., 15 : 937-946.

Dorigo U. \& Leboulanger C. 2001 - A PAM fluorescence-based method for assessing the effects of A photosystem II herbicides on freshwater periphyton. J. Appl. Phycol. $13: 463-534$.

Dorigo U. 2001. - Methodologies to assess the effects of pesticides on freshwater microphytobenthos. Tesi di Laurea. Université de Padoue, Italie : 112 p.

El Fantroussi S., Verschuere L., Verstraete W. \& Top E.M. 1999. Effect of phenylurea herbicides on soil microbial communities estimated by analysis of $16 \mathrm{~S}$ rRNA gene fingerprints and community-level physiological profiles. Appl. Environ. Microbiol., 65 : 982-988.

Engelen B., Meinken K., von Wintzingerode F., Heuer H., Malkomes P. \& Backhaus H. 1998. - Monitoring impact of a pesticide treatment on bacterial soil communities by metabolid and genetic fingerprinting in addition to conventional testing procedures. Appl. Environ. Microbiol., 64 : 2814-2821.
Faber M.J., Thompson D.G., Stephenson G.R. \& Boermans H.J. 1998. - Impact of glufosinate-ammonium and bialaphos on the phytoplankton community of a small eutrophic northern lake. Environ. Toxicol. Chem., $17:$ 1282-1290.

Foster P.L. 1982. - Metal resistances of chlorophyta from rivers polluted by heavy metals. Freshwater Biol., 12 : 41-61.

Goldsborough L.G. \& Robinson G. G. C. 1986. - Changes in periphytic algal community structure as a consequence of short herbicide exposures. Hydrobiologia, $138: 177-192$.

Guasch H., Admiraal W., Blanck H., Ivorra N., Lehmann V., Paulsson M., Real M. \& Sabater S. 1999. - Use of lotic periphyton communities as indicators of sensitivity to certain toxicants. In Prigiel J., Whitton B.A., Bukowska J. (eds). Use of algae for monitoring rivers III. Agence de l'Eau Artois-Picardie, Douai, France : 245-253.

Guasch H., Munoz I., Rosés N. \& Sabater S. 1997. - Changes in atrazine toxicity throughout succession of stream periphyton. $J$. Appl. Phycol., $9:$ 137-146.

Guasch H. \& Sabater S. 1998. - Light history influences the sensitivity to atrazine in periphytic algae. J. Phycol., $34: 233-241$.

Gustavson K., Petersen S., Pedersen B., Stuer-Lauridsen F., Pedersen S. \& Wängberg S.-A. 1999. - Pollution-Induced Community Tolerance (PICT) in coastal phytoplankton communities exposed to copper. Hydrobiologia, 416: 125-138.

Gustavson K. \& Wängberg S.-A. 1995.- Tolerance induction and succession in microalgae communities exposed to copper and atrazine. Aquat. Toxicol., $32: 283-302$.

Hamala J.A. \& Kollig H.P. 1985. - The effects of atrazine on periphyton communities in controlled laboratory ecosystems. Chemosphere, $14: 1391-1408$.

Herman D., Kaushik N. K. \& Solomon K. R. 1986. — Impact of atrazine on periphyton in freshwater enclosures and some ecological consequences. Can. J. Fish. Aquat. Sci., 43 : 1917-1925.

Harrison W. G., Eppley R. W. \& Renger E. H. 1977. - Phytoplankton nitrogen metabolism, nitrogen budget, and observations on copper toxicity : controlled ecosystem pollution experiment. Bull. Mar. Sci., $27: 44-57$.

Hersh C. \& Crumpton W.G. 1989. - Atrazine tolerance of algae isolated from two agricultural streams. Environ. Toxicol. Chem., $8: 327-332$.

Jurgensen T.A. \& Hoagland K.D. 1990. - Effects of short-term pulses of atrazine on attached algal communities in a small stream. Arch. Environ. Cont. Toxicol., 19 : 617-623.

Kasai F., Takamura N. \& Hatakeyama S. 1993. - Effects of simetryne on growth of various freshwater algal taxa. Environ. Poll., $79: 77-83$

Kasai F. 1999. - Shifts in herbicide tolerance in paddy field periphyton following herbicide application. Chemosphere, 38 : 919 931.

Knauer K., Behra R. \& Hemon H. 1999. - Toxicity of inorganic and methylated arsenic to algal communities from lakes along an arsenic contamination gradient. Aquat. Toxicol., $46: 223-232$.

Kosinsky R.J. 1984. - The effect of terrestrial herbicides on the community structure of stream periphyton. Environ. Poll., 36 : 165-189.

Lakshminarayana J.S.S., O'Neill H.J., Jonnavithula S.D., Léger D.A. \& Milburn P.H. 1992. — Impact of atrazine-bearing agricultural tile drainage discharge on planktonic drift of a natural stream. Environ. Pollut., 76 : 201-210.

Lampert W., Fleckner W., Pott E., Schober U. \& Störkel K. U. 1989. - Herbicide effects on planktonic systems of different complexity. Hydrobiologia, 188/189:415-424.

Loez C.R., Topalian M.L: \& Salibian A. 1995. - Effects of zinc on the structure and growth dynamics of a natural freshwater phytplankton assemblage reared in the laboratory. Environ. Poll., 88 : 275-281. 
Luoma S.N. 1977. - Detection of trace contaminant effects in aquatic ecosystems. J. Fish. Res. Board Can., 34 : 436-439.

Mayashich J.M., Karlander E.P. \& Terlizzi D.E. 1987.— Growth responses of Nannochloris occulata Droop and Phaeodactylum tricornutum Bohlin to the herbicide atrazine as influenced by light intensity and temperature in unialgal and bialgal assemblage. Aquat. Toxicol., $10: 187-197$.

McCaig, A.E., Glover A.L. \& Prosser J.I. 1999. - Molecular analysis of bacterial community structure and diversity in unimproved and improved grass pastures. Appl. Environ. Microbiol., 65 : 1721-1730.

McCormick P.V. \& Cairns, J. Jr.1994. - Algae as indicators of environmental change. J. Appl. Phycol., 6 : 509-526.

Millie D.F., Hersh C.M. \& Dionigi C.P. 1992. - Simazine -induced inhibition in photoacclimated populations of Anabaena circinalis (Cyanophyta). J. Phycol., 28 : 19-26.

Millward R.N. \& Grant A. 1995. - Assessing the impact of copper on nematode communities from a chronically metal-enriched estuary using Pollution-Induced Community Tolerance. Mar. Poll. Bull., 30 : 701-706.

Molander S., Dahl B., Blanck H., Jonsson J. \& Sjöström M. 1992. Combined effects of tri-n-butyltin (TBT) and diuron on marine periphyton communities detected as Pollution-Induced Community Tolerance. Arch. Environ. Contam. Toxicol., 22 : 419-427.

Molander S. \& Blanck H. 1992. - Detection of pollution-induced community tolerance (PICT) in marine periphyton communities established under diuron exposure. Aquat. Toxicol., 22 : 129-144.

Molander S., Blanck H. \& Söderström M. 1990. - Toxicity assessment by pollution-induced community tolerance (PICT), and identification of metabolites in periphyton communities after exposure to 4,5,6-trichloroguaiacol. Aquat. Toxicol., 18 : 115-136.

Molander S. 1991. - Detection, validity and specificity of pollution-induced community tolerance (PICT). Thèse de Doctorat, Université de Göteborg, Suède : $30 \mathrm{p}$.

Munawar M., Munawar I.F. \& Mayfield C.I. 1987. - Differential sensitivity of natural phytoplankton size assemblages to metal mixture toxicity. Arch. Hydrobiol., 25 : 123-139.

Murray A.E., Hollibraugh J.T. \& Orrego C. 1996. - Phylogenetic compositions of bacterioplankton from two California estuaries compared by denaturing gradient gel electrophoresis of $16 \mathrm{~S} \mathrm{rD-}$ NA fragments. Appl. Environm. Microbiol., $62: 2676-2680$.

Niederlehner B.R. \& Cairns J. Jr. 1992. - Community response to cumulative toxic impact : effects of acclimation on zinc tolerance of Aufwuchs. Can. J. Fish. Aquat. Sci., $49: 2155-2163$.

de Noyelles F., Kettle W.D. \& Sinn D. 1982. - The response of plankton communities in experimental ponds to atrazine, the most heavily used pesticide in the United States. Ecology, $63: 1285-$ 1293.

Nyström B.A., Becker-van Slooten K., Bérard A., Grandjean D., Druart J.C. \& Leboulanger C. 2001 - Toxic effects of Irgarol 1051 on phytoplankton communities and macrophytes in Lake Geneva. Water Res., sous presse.

Nyström B.A. 1997. - Metabolic indicators of ecotoxicological effects in freshwater periphyton communities. Thèse de Doctorat, Université de Göteborg, Suède : $26 \mathrm{p}$.

Nyström B., Paulsson M., Almgren K. \& Blanck H. 2000. - Evaluation of the capacity form development of atrazine tolerance in periphyton from a Swedish freshwater site as determined by inhibition of photosynthesis and sulfolipid synthesis. Environ. Toxicol. Chem., 19 :1324-1331

Nyström B., Björnsäter B. \& Blanck H. 1999. - Effects of sulfonylurea herbicides on non-target aquatic micro-organisms - Growth inhibition of micro-algae and short-term inhibition of adenine and thymidine incorporation in periphyton communities. Aquat. Toxicol., $47: 9-22$.
Paulsson M., Nyström B. \& Blanck H., 2000. — Long-term toxicity of zinc to bacteria and algae in periphyton communities from the river Göta flv, based on a microcosm study. Aquat. Toxicol., 47 : 243-257.

Pascoe D., Wenzel A., Janssen C., Girling A.E., Jüttner I., Fliedner A., Blockwell S.J., Maund S.J., Taylor E.J., Diedrich M., Persoone G., Verhelst P., Stephenson R.R., Crossland N.O., Mitchell G.C., Pearson P., Tattersfield L., Lay J.-P., Peither A., Neumeier B. \& Velletti A.-R. 2000. - The development of toxicity tests for freshwater polluants and their validation in stream and pond mesocosms. Water Res., 34 : 2323-2329.

Payne C.D. \& Price N.M. 1999. ( Effects of cadmium toxicity on growth and elemental composition of marine phytoplankton. $J$. Phycol., 35 : 293-302.

Pérès F., Coste M., Ribeyre F., Ricard M. \& Boudou A. 1997. - Effects of methylmercury and inorganic mercury on periphytic diatom communities in freshwater indoor microcosms. J. Appl. Phycol., $9: 215-227$.

Petersen S. \& Gustavson K. 1998. - Toxic effects of tri-butyl-tin (TBT) on autotrophic pico- nano-, and microplankton assessed by size fractionated pollution-induced community tolerance (SFPICT) concept. Aquat. Toxicol., 40 : 253-264.

Ramirez Torres A.M. \& O'Flaherty L.M. 1976. - Influence of pesticides on Chlorella, Chlorococcum, Stigeoclonium (Chlorophyceae), Tribonema, Vaucheria (Xanthophyceae) and Oscillatoria (Cyanophyceae). Phycologia, $15: 25-36$.

Ruth P. 1978. - Effects of trace metals in the aquatic ecosystem. Amer. Sci., $66: 191$.

Saint Olive A., Leboulanger C. \& Bérard A. 2001. - Utilisation des communautés de microalgues telluriques comme bioindicateurs potentiels de contamination des sols. Premiers essais sur l'extraction des algues, la mesure de leur activité photosynthétique et leur sensibilité à l'atrazine. C.R. XXXIème Congrès du Groupe français des Pesticides, Lyon : 15-17 mai $2001: 80$.

Seguin F., Le Bihan F., Leboulanger C. \& Bérard A. sous presse. Risk asessment and of pollution : induction of atrazine tolerance in phytoplankton communities in freshwater outdoor mesocosms, using chlorophyll fluorescence as endpoint . Water Res.

Seguin F., Le Bihan F., Leboulanger C. \& Bérard A. 2000. - Evaluation du risque écotoxicologique en systèmes expérimentaux : Impacts de l'atrazine sur la tolérance des communautés phytoplanctoniques en mésocosmes lentiques. CILEF 2000 ClermontFerrand, 16-21 juillet 2000.

Slauenwhite D.E., Wangersky P.J. \& Johnson B.D. 1991. — Effect of a spring phytoplankton bloom on dissolved copper speciation in Bedford Basin. EMECS, $9023: 137-144$.

Soldo D.\& Berha R. 2000. ( Long-term effects of copper on the structure of freshwater periphyton communities and their tolerance to copper, zinc, nickel and silver. Aquat. Toxicol., $47: 181-189$.

Sommer U. 1986. - Phytoplankton competition along a gradient of dilution rates. Oecologia, $68: 503-506$.

Stay F.S., Katko A., Rohm C.M., Fix M.A. \& Larsen D.P. 1989. The effects of atrazine on microcosms developed from four natural plankton communities. Arch. Environ. Contam. Toxicol., 18 : 866-875.

Takamura N., Kasai F. \& Watanabe M.M. 1989. (Effects of Cu, Cd and $\mathrm{Zn}$ on photosynthesis of freshwater benthic algae. J. Appl. Phycol., 1 : 39-52.

Takamura N., Kasai F. \& Watanabe M.M. 1990. ( Unique response of cyanophyceae to copper. J. Appl. Phycol., $2: 293-296$.

Taub F.B., Kindig A.C., Meador J.P. \& Swartzman G.L. 1991. ( Effects of «seasonal succession» and grazing on copper toxicity in aquatic microcosms. Verh. Internat. Verein. Limnol., 24 : 2205 2214. 
Tilman D. 1977. - Resource competition between planktonic algae: an experimental and theoretical approach. Ecology, 58 : 338-348.

Vindimian E. \& Garric J. 1993. - Bio-essais et bio-indicateurs de toxicité dans le milieu naturel. «Etude Inter-Agences $\mathrm{n}^{\circ} 17: 54 \mathrm{p}$.

Wängberg S.-A 1995. - Effects of arsenate and copper on the algal communities in polluted lakes in the northern parts of Sweden assayed by PICT (Pollution-Induced Community Tolerance). $\mathrm{Hy}$ drobiologia, $306:$ 109-124.

Wängberg S.-A. \& Blanck H. 1990 - Arsenate tolerance in marine periphyton communities under different nutrient regimes. J. Exp. Mar. Biol. Ecol., 139 : 119-134.
Wängberg S.-A., Heyman U. \& Blanck H. 1991 - Long-term and short-term arsenate toxicity to freshwater phytoplankton and periphyton in limnocorrals. Can. J. Fish. Aquat. Sci., 48 : 173-182.

Wängberg S.-A. 1989 - Arsenate tolerance in periphyton and pytoplankton. Thèse de Doctorat, Université de Göteborg, Suède : $31 \mathrm{p}$.

Winner R. W. \& Owen H.A. 1991. — Seasonal variability in the sensitivity of freshwater phytoplankton communities to a chronic copper stress. Aquat. Toxicol., $19: 73-88$.

Wolfe J.M. \& Rice E.L. 1979. - Allelopathic interactions among algae. J. Chem. Ecol., 5 : 533-542. 